BMJ Open

Diabetes

Research

\& Care

\title{
Joint associations of metabolically healthy abdominal obesity and non- alcoholic fatty liver disease with prediabetes and diabetes in Chinese adults
}

\author{
Junfeng Zhang, ${ }^{1}$ Qiaoyan Xu, ${ }^{1}$ Fangfang Lai, ${ }^{1}$ Ning Chen, ${ }^{2}$ Mingzhu Lin, ${ }^{3}$ \\ Yongwen Liu, ${ }^{4}$ Wei Zhang, ${ }^{5}$ Changqin Liu (D) ${ }^{3}$ Shunqin Wang, ${ }^{6}$ Zhibin Li (D) ${ }^{7}$
}

To cite: Zhang J, Xu Q, Lai F, et al. Joint associations of metabolically healthy abdominal obesity and non-alcoholic fatty liver disease with prediabetes and diabetes in Chinese adults. BMJ Open Diab Res Care 2021;9:e002362. doi:10.1136/ bmjdrc-2021-002362

JZ, QX, FL and NC contributed equally.

Received 2 May 2021

Accepted 31 July 2021
Check for updates

(C) Author(s) (or their employer(s)) 2021. Re-use permitted under CC BY-NC. No commercial re-use. See rights and permissions. Published by BMJ.

For numbered affiliations see end of article.

Correspondence to Dr Zhibin Li; zhibinli33@hotmail.com and Dr Shunqin Wang; 21831168@qq.com

\section{ABSTRACT}

Introduction We aimed to evaluate the joint associations of metabolically healthy abdominal obesity (MHAO) with non-alcoholic fatty liver disease (NAFLD) on risks of diabetes and prediabetes.

Research design and methods Baseline information of 1318 adults with abdominal obesity (waist circumference $\geq 90 \mathrm{~cm}$ for men and $80 \mathrm{~cm}$ for women) from an ongoing cohort study in Xiamen, China were analyzed. Metabolic health was identified as none of the criteria of metabolism syndrome, except for obesity, was met. Results MHAO and metabolically unhealthy abdominal obesity (MUA0) were identified on 173 (13.1\%) and $1145(86.9 \%)$ subjects. NAFLD was further diagnosed on $60(34.7 \%)$ in MHAO and 721 (63.0\%) in MUAO groups $(p<0.001)$. Both MUAO (vs MHAO) and NAFLD (vs nonNAFLD) were independently associated with increased risks of diabetes as well as prediabetes plus diabetes, with the adjusted ORs (95\% Cls) of 9.40 (3.38 to 26.14 ) and 2.02 (1.47 to 2.77), respectively. Compared with MHAO and non-NAFLD, MHAO and NAFLD showed significantly increased risks of prediabetes plus diabetes with the adjusted ORs ( $95 \%$ Cls) of 2.87 (1.32 to $6.27, \mathrm{p}=0.008$ ). And there were significantly positive trends between increasing categories jointly by MHAO and NAFLD (from MHAO and non-NAFLD, MHAO and NAFLD, MUAO and nonNAFLD to MUAO and NAFLD) with risks of diabetes and prediabetes plus diabetes (both trend tests: $p<0.001$ ). Conclusions About $35 \%$ of subjects with MHAO accompanied by NAFLD showed excessive risk of prediabetes plus diabetes compared with MHAO and nonNAFLD. Thus, NAFLD should be screened and intervened even for those subjects with metabolically healthy obesity (MHO) and should be considered as one additional criterion when defining and diagnosing $\mathrm{MHO}$.

\section{INTRODUCTION}

The global prevalence of diabetes has quadrupled during the past three decades, which results in a heavy public health burden worldwide. ${ }^{1}$ The International Diabetes Federation estimated around $10.2 \%$ and $10.9 \%$ adults worldwide will have diabetes in 2030 and

\section{Significance of this study}

What is already known about this subject?

- Ideas, definitions and diagnosis criteria of metabolically healthy obesity (MHO) are in debating, and non-alcoholic fatty liver disease (NAFLD) has not been considered in current definition and diagnosis criteria of $\mathrm{MHO}$.

What are the new findings?

- About $35 \%$ of subjects with metabolically healthy abdominal obesity (MHAO) who were further diagnosed with NAFLD had increased risk of prediabetes plus diabetes compared with those with MHAO and non-NAFLD.

- Both metabolically unhealthy abdominal obesity (MUAO) (vs MHAO) and NAFLD (vs non-NAFLD) were independently associated with increased risks of diabetes as well as prediabetes plus diabetes.

- There were significantly positive trends between increasing categories jointly by MUAO and NAFLD (from MHAO and non-NAFLD, MHAO and NAFLD, MUAO and non-NAFLD to MUAO and NAFLD) with risks of diabetes and prediabetes plus diabetes.

How might these results change the focus of research or clinical practice?

- NAFLD should be screened and intervened for subjects with $\mathrm{MHO}$ and should be considered as one additional criterion when defining and diagnosing $\mathrm{MHO}$

2045, respectively. ${ }^{2}$ China has been experiencing one of the rapidest growths of diabetes incidence during the past 30 years. According to a nationally representative data from 2015 to 2017 in mainland China, the weighted prevalence rates of total diabetes were $12.8 \%$ $(95 \%$ CI $12.0 \%$ to $13.6 \%)$ and $11.2 \%(95 \%$ CI $10.5 \%$ to $11.9 \%$ ) based on the American Diabetes Association (ADA) and WHO criteria, respectively. ${ }^{3}$ Obesity represents 
another severe global public health problem due to its explosively rapid increase in prevalence and consequences on dramatically increased morality from noncommunicable diseases, such as cardiovascular diseases, type 2 diabetes and certain types of cancer. ${ }^{4-6}$ Studies have documented that there is a subgroup of individuals with obesity who are devoid of obesity-related metabolic complications, such as diabetes and atherosclerosis, which arises the concept of metabolically healthy obesity (MHO) since $1950 .^{7-10}$ Since there is no consensus available on gold standard criteria for MHO, evidence on the association of $\mathrm{MHO}$ and diabetes is limited and controversial. ${ }^{11}$ Some meta-analyses demonstrated that MHO was associated with a significantly lower incidence of type 2 diabetes compared with metabolically unhealthy obese (MUO) and a substantially increased risk of developing type 2 diabetes compared with metabolically healthy normal weight. ${ }^{12}$

Obesity is a condition characterized by the excessive accumulation and storage of fat in human body, mainly in limbs, viscera and liver. Body mass index (BMI) and waist circumference (WC) are widely used as indices of general and abdominal obesity, respectively; meanwhile excessive fat accumulation in liver has not been considered when defining obesity. Non-alcoholic fatty liver disease (NAFLD) typically comprises a spectrum of pathological conditions including simple steatosis, non-alcoholic steatohepatisis and cirrhosis due to significant fat accumulation in the liver. ${ }^{13}$ NAFLD is a kind of chronic liver disease and contributes to extrahepatic diseases, such as type 2 diabetes and cardiovascular disease. ${ }^{13} 14$ NAFLD has been consistently shown to be associated with metabolic/insulin resistance (IR) syndrome and should be included in the definition of metabolic syndrome, which may therefore predict diabetes. ${ }^{15}{ }^{16}$ Although NAFLD itself has been shown to predict the transition from MHO to MUO,${ }^{17}$ NAFLD is seldom considered as one criterion when defining and diagnosing $\mathrm{MHO}$, and little evidence on prevalence of NAFLD in those with MHO is available. Moreover, there is no evidence currently available about the integrated effects of NAFLD with MHO on risks of diabetes and prediabetes. Therefore, in the present study with 1318 community-living Chinese adults with abdominal obesity, we aimed to evaluate the independent associations of metabolically healthy abdominal obesity (MHAO) and NAFLD separately and jointly on risks of diabetes and prediabetes plus diabetes.

\section{RESEARCH DESIGN AND METHODS}

\section{Study design and subjects}

Details on study design and subjects recruitment have been described previously. ${ }^{18}{ }^{19}$ Briefly, 1523 communityliving healthy adults aged 40 years or older with abdominal obesity (WC $>90 \mathrm{~cm}$ for men and $80 \mathrm{~cm}$ for women) living in Lianqian community, Xiamen, China were recruited as baseline of the cohort study in 2011. Of them, 205 had incomplete data on clinical, biochemical or hepatic ultrasonography scanning measurements; then $1318(86.5 \%)$ subjects with the complete data were left for the present analysis.

\section{Measurements}

Details on methods of subject sampling and evaluation, including face-to-face interviews and clinical characteristics measurements, have been described previously. ${ }^{18} 19$ Blood samples were obtained after 12-hour fasting and tested in the central laboratory of the First Affiliated Hospital, Xiamen University. Plasma glucose, liver enzymes and serum lipid profiles, including triglyceride (TG), total cholesterol (TC) and high-density lipoproteincholesterol (HDL-C) were determined on a HITACHI 7450 analyzer (HITACHI, Tokyo, Japan). Homeostasis model assessment-insulin resistance (HOMA-IR) was calculated using the formula: fasting serum insulin $(\mathrm{mU} / \mathrm{L}) \times$ fasting plasma glucose $(\mathrm{FPG})(\mathrm{mmol} / \mathrm{L}) / 22.5$. And IR was defined as HOMA-IR $\geq 2.6 \times 10^{-6} \mathrm{~mol} \times \mathrm{IU} /$ $\mathrm{L}^{2}{ }^{20}$ Hepatic ultrasonography scanning and diagnosis of hepatic steatosis have been described previously ${ }^{18} 19$ and followed the guidelines for the diagnosis and treatment of NAFLDs in China (Chinese National Consensus Workshop on NAFLD) ${ }^{21}$

\section{Hepatic steatosis indices}

Fatty liver index (FLI) is a non-invasive method of assessing hepatic steatosis and is calculated based on laboratory and anthropometric measures, including TG, gamma-glutamyl transpeptidase (GGT), BMI and WC. The FLI was calculated by the following formula: $\mathrm{FLI}=\left(\mathrm{e}^{0.953 \times \ln (\mathrm{TG})+0.139 \times \mathrm{BMI}+0.718 \times \ln }\right.$

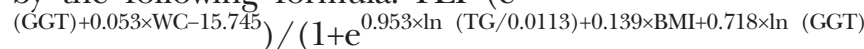
$+0.053 \times \mathrm{WC}-15.745) \times 100 .^{22}$ Hepatic steatosis index $(\mathrm{HSI})=8 \times$ alanine aminotransferase/aspartate transaminase+BMI $(+2$, if diabetes mellitus; +2 , if female) ${ }^{23}$

\section{Definition of metabolically healthy abdominal obesity}

Abdominal obesity was defined as $\mathrm{WC} \geq 90 \mathrm{~cm}$ for men and $80 \mathrm{~cm}$ for women. ${ }^{24}$ All subjects in the present study had abdominal obesity which was considered as one of the recruitment criteria. Subjects were diagnosed as being metabolically healthy if none of the following criteria was met: (1) systolic blood pressure (BP) $\geq 130$ or diastolic $\mathrm{BP} \geq 85 \mathrm{~mm} \mathrm{Hg}$; (2) $\mathrm{FPG} \geq 100 \mathrm{mg} / \mathrm{dL}(5.6 \mathrm{mmol} / \mathrm{L})$; (3) $\mathrm{TG} \geq 150 \mathrm{mg} / \mathrm{dL}(1.7 \mathrm{mmol} / \mathrm{L}) ;(4) \mathrm{HDL}-\mathrm{C}<40 \mathrm{mg} / \mathrm{dL}$ $(1.03 \mathrm{mmol} / \mathrm{L})$ in men and $<50 \mathrm{mg} / \mathrm{dL}(1.30 \mathrm{mmol} / \mathrm{L})$ in women. ${ }^{25}{ }^{26}$ Otherwise, subjects were defined as being metabolically unhealthy if one or more of the above criteria was met. Therefore, all subjects in the present study were dichotomized as either MHAO or metabolically unhealthy abdominal obesity (MUAO).

\section{Definitions of diabetes and prediabetes}

According to ADA 2020 criteria, diabetes was defined as (1) a self-reported history of diabetes previously diagnosed by healthcare professionals; (2) FGP $\geq 126 \mathrm{mg}$ / dL (7.0 mmol/L); (3) 2-hour plasma glucose (2-h PG, oral glucose tolerance test $($ OGTT) $) \geq 200 \mathrm{mg} / \mathrm{dL}$ $(11.1 \mathrm{mmol} / \mathrm{L})$ or $(4)$ hemoglobin A1c $(\mathrm{HbAlc}) \geq 6.5 \%$. 
Prediabetes were defined as (1) FPG levels between 100 $\mathrm{mg} / \mathrm{dL}(5.6 \mathrm{mmol} / \mathrm{L})$ and $125 \mathrm{mg} / \mathrm{dL}(6.9 \mathrm{mmol} / \mathrm{L}),(2)$ 2-h PG levels between $140 \mathrm{mg} / \mathrm{dL}(7.8 \mathrm{mmol} / \mathrm{L})$ and 199 $\mathrm{mg} / \mathrm{dL}(11.0 \mathrm{mmol} / \mathrm{L})$ or (3) HbAlc between $5.7 \%$ and $6.4 \%$ in participants without a prior diabetes diagnosis. ${ }^{27}$

\section{Statistical analyses}

Data were presented as the mean $\pm \mathrm{SD}$ for continuous variables or number and percentage for categorical variables. Skewness and kurtosis tests for continuous variables were conducted and found them followed approximation of normal distributions. Differences between subjects categorized by MHAO and NAFLD were analyzed using oneway analysis of variance for continuous variables and $\chi^{2}$ test for categorical variables. Bar graphs showing prevalence rates of diabetes, prediabetes and normal glucose test were made across abdominal obesity and NAFLD (MHAO and non-NAFLD, MHAO and NAFLD, MUAO and non-NAFLD, MUAO and NAFLD, respectively).

Multivariable logistic regression models were used to calculate the adjusted ORs and 95\% CIs of abdominal obesity (MUAO vs MHAO) and NAFLD (yes vs no) separately and jointly in different models with adjustment for potential confounders for diabetes and prediabetes plus diabetes, separately. In model 1 , age and sex were adjusted for; in model 2, educational level, smoking and drinking habits and regular physical exercise plus model 1 were adjusted for; in model 3, BMI, systolic and diastolic BP, TG, TC, HDL-C and low-density lipoproteincholesterol (LDL-C) and serum uric acid plus model 2 were adjusted for. Additonally, hepatic steatosis indices (FLI and HIS) were analyzed separately in the same models as above. All $\mathrm{p}$ values were two-sided and $\mathrm{p}$ value $<0.05$ was considered statistically significant. All statistical analyses were performed using Stata V.14.0 (StataCorp, College Station, Texas, USA).

\section{RESULTS}

Demographic and clinical characteristics stratified jointly by MHAO and NAFLD

Among the 1318 subjects with abdominal obesity, 924 $(70.1 \%)$ were women and $394(29.9 \%)$ were men. The means $( \pm$ SD) for women and men were $92.1( \pm 6.8)$ and $97.0( \pm 6.3) \mathrm{cm}(\mathrm{p}<0.001)$ for $\mathrm{WC}$ and were $53.4( \pm 6.8)$ and $53.3( \pm 7.3)$ years for age $(\mathrm{p}=0.808)$, respectively. Of them, $173(13.1 \%)$ and $1145(86.9 \%)$ were identified as MHAO and MUAO, respectively. NAFLD were further diagnosed for $60(34.7 \%)$ in those with MHAO and 721 $(63.0 \%)$ in those with MUAO $(\mathrm{p}<0.001)$.

Differences of demographics, lifestyle habits and clinical characteristics stratified jointly by MHAO and NAFLD are shown in table 1 . Generally, with increasing categories jointly by MHAO and NAFLD (from MHAO and non-NAFLD, MHAO and NAFLD, MUAO and nonNAFLD to MUAO and NAFLD), subjects were more likely to be male, older and had higher levels of indices of obesity (BMI, WC), systolic and diastolic BP, TG, TC,
FPG, 2-h PG, HbA1c, fasting insulin, HOMA-IR, serum uric acid, hepatic steatosis indices (FLI and HIS), prevalence of IR and significantly lower level of education and HDL-C.

Prevalence rates of diabetes and prediabetes stratified jointly by MHAO and NAFLD

Diabetes and prediabetes were identified on 345 (26.2\%) and $803(60.9 \%)$ subjects, respectively. Stratified jointly by MHAO and NAFLD, the prevalence rates of diabetes were $0.9 \%, 5.0 \%, 18.2 \%$ and $36.6 \%$ for those with MHAO and non-NAFLD, MHAO and NAFLD, MUAO and nonNAFLD to MUAO and NAFLD, respectively (trend test: $\mathrm{p}<0.001$ ); and the prevalence rates of prediabetes plus diabetes were $54.9 \%, 80.0 \%, 86.3 \%$ and $93.2 \%$, respectively (trend test: $\mathrm{p}<0.001$ ) (table 1 and figure 1 ).

\section{Joint associations of MHAO and NAFLD with diabetes}

Table 2 shows the adjusted ORs with associated 95\% CIs of MHAO and NAFLD separately and jointly for diabetes by using the multivariable logistic regression analyses with adjustment for potential confounding factors in different models. In model 1, both MUAO (vs MHAO) and NAFLD (yes vs no) showed significantly increased risk of diabetes, and the adjusted ORs (95\% CIs) were 15.90 (5.84 to 43.31, $\mathrm{p}<0.001$ ) and 2.95 (2.21 to 3.92, $\mathrm{p}<0.001)$, respectively. Increasing categories jointly by MHAO and NAFLD (from MHAO and non-NAFLD, MHAO and NAFLD, MUAO and non-NAFLD to MUAO and NAFLD) showed a significantly positive trend of increased risk of diabetes (trend test: $\mathrm{p}<0.001$ ). With the category of MHAO and non-NAFLD as the reference, those with MUAO and non-NAFLD and MUAO and NAFLD both showed significantly increased risks of diabetes with the adjusted ORs (95\% CIs) of 22.34 (3.07 to 162.70 ) and 57.49 (7.97 to 414.93) (both $\mathrm{p}$ values $<0.001$ ), but those with MHAO and NAFLD did not show significantly increased risk of diabetes (OR (95\% CI) 5.68 ( 0.58 to 55.96), $\mathrm{p}=0.136$ ). In model 2 and model 3 with further adjustment for other potential confounding factors (educational level, ever smoking, ever drinking, regular physical exercise habits and BMI, systolic and diastolic BP, TG, HDL-C and LDL-C and serum uric acid, respectively), all the results were quite similar to those in model 1 and did not change much. For all subjects, hepatic steatosis indices, both FLI and HIS, were significantly associated with risk of diabetes with adjusted ORs (95\% CIs) of 1.02 (1.01 to 1.03 ) and 1.22 (1.18 to 1.27 ), respectively (both $\mathrm{p}$ values $<0.001$, model 3 ). Stratified analyses showed that, for both MHAO and subjects with MUAO, higher FLI and HIS were significantly associated with increased risk of diabetes (model 3).

Joint associations of MHAO and NAFLD with prediabetes plus diabetes

Table 2 shows both MUAO and NAFLD, compared with MHAO and non-NAFLD, were significantly associated with increased risks of prediabetes plus diabetes in model 
Table 1 Demographic, lifestyle and clinical characteristics of 1318 subjects stratified by MHAO and NAFLD

\begin{tabular}{|c|c|c|c|c|c|}
\hline \multirow[b]{2}{*}{ Variables } & \multicolumn{2}{|c|}{ MHAO (n=173, 13.1\%) } & \multicolumn{2}{|c|}{ MUAO (n=1145, 86.9\%) } & \multirow[b]{2}{*}{$P$ value } \\
\hline & Non-NAFLD & NAFLD & Non-NAFLD & NAFLD & \\
\hline \multicolumn{6}{|l|}{ Demographics } \\
\hline N (\%) & $113(8.6 \%)$ & $60(4.5 \%)$ & $424(32.2 \%)$ & $721(54.7 \%)$ & \\
\hline Sex & & & & & $<0.001 \dagger$ \\
\hline Female (n, \%) & $94(83.2 \%)$ & $46(76.7 \%)$ & 332 (78.3\%) & $452(62.7 \%)$ & \\
\hline Male (n, \%) & $19(16.8 \%)$ & $14(23.3 \%)$ & $92(21.7 \%)$ & 269 (37.3\%) & \\
\hline Age (years) & $50.4 \pm 6.7$ & $51.5 \pm 6.6$ & $53.3 \pm 6.9$ & $54.1 \pm 7.0$ & $<0.001 \dagger$ \\
\hline Education categories, (n, \%) & & & & & $0.024^{*}$ \\
\hline Illiteracy & $19(16.8 \%)$ & $12(20.0 \%)$ & 144 (34.0\%) & $196(27.2 \%)$ & \\
\hline Elementary school & $40(35.4 \%)$ & $18(30.0 \%)$ & 125 (29.5\%) & 206 (28.6\%) & \\
\hline Middle school & $25(22.1 \%)$ & $15(25.0 \%)$ & $90(21.2 \%)$ & $170(23.6 \%)$ & \\
\hline High school or above & $19(16.8 \%)$ & $8(13.3 \%)$ & 42 (9.9\%) & $102(14.1 \%)$ & \\
\hline College & $10(8.9 \%)$ & $7(11.7 \%)$ & $23(5.4 \%)$ & 47 (6.5\%) & \\
\hline \multicolumn{6}{|l|}{ Lifestyle } \\
\hline Ever smoking (n, \%) & $24(21.2 \%)$ & $10(16.7 \%)$ & $82(19.3 \%)$ & $224(31.1 \%)$ & $<0.001 \dagger$ \\
\hline Ever drinking (n, \%) & $16(14.2 \%)$ & $7(11.7 \%)$ & $52(12.3 \%)$ & $126(17.5 \%)$ & 0.094 \\
\hline Regular physical exercise (n, \%) & $39(34.5 \%)$ & $19(31.7 \%)$ & 149 (35.1\%) & $225(31.2 \%)$ & 0.558 \\
\hline \multicolumn{6}{|l|}{ Clinical characteristics } \\
\hline BMI $\left(\mathrm{kg} / \mathrm{m}^{2}\right)$ & $26.2 \pm 2.1$ & $27.4 \pm 3.4$ & $26.3 \pm 2.5$ & $28.3 \pm 3.1$ & $<0.001 \dagger$ \\
\hline Waist circumference (cm) & $90.5 \pm 5.6$ & $92.4 \pm 6.7$ & $90.9 \pm 5.6$ & $95.7 \pm 7.3$ & $<0.001 \dagger$ \\
\hline Body fat rate (\%) & $34.6 \pm 5.7$ & $35.5 \pm 7.0$ & $34.3 \pm 6.1$ & $35.1 \pm 7.3$ & 0.169 \\
\hline Systolic blood pressure $(\mathrm{mm} \mathrm{Hg})$ & $117.2 \pm 7.9$ & $117.1 \pm 7.1$ & $132.8 \pm 17.3$ & $138.0 \pm 16.7$ & $<0.001 \dagger$ \\
\hline Diastolic blood pressure $(\mathrm{mm} \mathrm{Hg})$ & $70.7 \pm 6.8$ & $71.5 \pm 5.7$ & $78.6 \pm 10.4$ & $82.2 \pm 10.4$ & $<0.001 \dagger$ \\
\hline Triglyceride (mmol/L) & $0.96 \pm 0.34$ & $1.15 \pm 0.30$ & $1.53 \pm 1.08$ & $2.26 \pm 1.38$ & $<0.001 \dagger$ \\
\hline Total cholesterol (mmol/L) & $5.62 \pm 0.87$ & $5.70 \pm 0.79$ & $5.77 \pm 1.11$ & $6.00 \pm 1.13$ & $<0.001 \dagger$ \\
\hline HDL-cholesterol (mmol/L) & $1.60 \pm 0.27$ & $1.55 \pm 0.31$ & $1.43 \pm 0.31$ & $1.29 \pm 0.26$ & $<0.001 \dagger$ \\
\hline LDL-cholesterol (mmol/L) & $3.58 \pm 0.77$ & $3.63 \pm 0.70$ & $3.64 \pm 1.00$ & $3.69 \pm 1.07$ & 0.634 \\
\hline Fasting plasma glucose (mmol/L) & $5.21 \pm 0.25$ & $5.18 \pm 0.27$ & $5.96 \pm 1.13$ & $6.49 \pm 2.06$ & $<0.001 \dagger$ \\
\hline 2-h PG (OGTT, mmol/L) & $6.55 \pm 1.28$ & $7.29 \pm 1.42$ & $8.15 \pm 3.13$ & $10.09 \pm 4.53$ & $<0.001 \dagger$ \\
\hline $\mathrm{HbA1c}(\%)$ & $5.75 \pm 0.31$ & $5.84 \pm 0.28$ & $6.00 \pm 0.69$ & $6.43 \pm 1.23$ & $<0.001 \dagger$ \\
\hline Fasting insulin (mlU/L) & $8.7 \pm 3.5$ & $10.7 \pm 5.3$ & $10.6 \pm 5.9$ & $14.6 \pm 7.4$ & $<0.001 \dagger$ \\
\hline HOMA-IR $\left(\times 10^{-6} \mathrm{~mol} \times \mathrm{IU} / \mathrm{L}^{2}\right)$ & $2.02 \pm 0.86$ & $2.45 \pm 1.21$ & $2.89 \pm 2.60$ & $4.23 \pm 2.66$ & $<0.001 \dagger$ \\
\hline $\operatorname{IR}(\mathrm{n}, \%)$ & $19(16.8 \%)$ & $22(36.7 \%)$ & $204(48.1 \%)$ & $536(74.3 \%)$ & $<0.001 \dagger$ \\
\hline Blood uric acid $(\mu \mathrm{mol} / \mathrm{L})$ & $310.7 \pm 84.8$ & $343.8 \pm 76.4$ & $337.3 \pm 84.1$ & $385.3 \pm 94.6$ & $<0.001 \dagger$ \\
\hline AST (U/L) & $22.0 \pm 5.4$ & $23.5 \pm 6.4$ & $24.2 \pm 7.7$ & $24.0 \pm 9.4$ & 0.683 \\
\hline $\mathrm{ALT}(\mathrm{U} / \mathrm{L})$ & $21.0 \pm 10.7$ & $26.4 \pm 15.1$ & $25.7 \pm 19.3$ & $28.8 \pm 19.0$ & 0.064 \\
\hline GGT (U/L) & $29.3 \pm 26.4$ & $27.9 \pm 14.3$ & $29.2 \pm 22.6$ & $42.3 \pm 29.2$ & $<0.001 \dagger$ \\
\hline FLI & $31.6 \pm 15.6$ & $42.1 \pm 19.7$ & $40.4 \pm 19.1$ & $64.1 \pm 19.9$ & $<0.001 \dagger$ \\
\hline $\mathrm{HSI}$ & $35.3 \pm 3.4$ & $37.7 \pm 4.9$ & $35.9 \pm 3.5$ & $39.6 \pm 4.7$ & $<0.001 \dagger$ \\
\hline Diabetes (n, \%) & $1(0.9 \%)$ & $3(5.0 \%)$ & $77(18.2 \%)$ & $264(36.6 \%)$ & $<0.001 \dagger$ \\
\hline Prediabetes (n, \%) & $61(54.0 \%)$ & $45(75.0 \%)$ & $289(68.2 \%)$ & $408(56.6)$ & $<0.001 \dagger$ \\
\hline Prediabetes plus diabetes (n, \%) & $62(54.9 \%)$ & $48(80.0 \%)$ & 366 (86.3\%) & 672 (93.2\%) & $<0.001 \dagger$ \\
\hline
\end{tabular}

All percentages are column percentage; except for percentages, all values are mean \pm SD.

${ }^{*} \mathrm{P}<0.05$.

$\dagger P<0.001$

ALT, alanine transaminase; AST, aspartate transaminase; BMI, body mass index; FLI, fatty liver index; GGT, gamma-glutamyl transpeptidase; HbA1c, hemoglobin A1c; HDL, high-density lipoprotein; HOMA, homeostasis model assessment; 2-h PG, 2-hour plasma glucose; HSI, hepatic steatosis index; IR, insulin resistance; LDL, low-density lipoprotein-cholesterol; MHAO, metabolically healthy abdominal obesity; MUAO, metabolically unhealthy abdominal obesity; NAFLD, non-alcoholic fatty liver disease; OGTT, oral glucose tolerance test. 


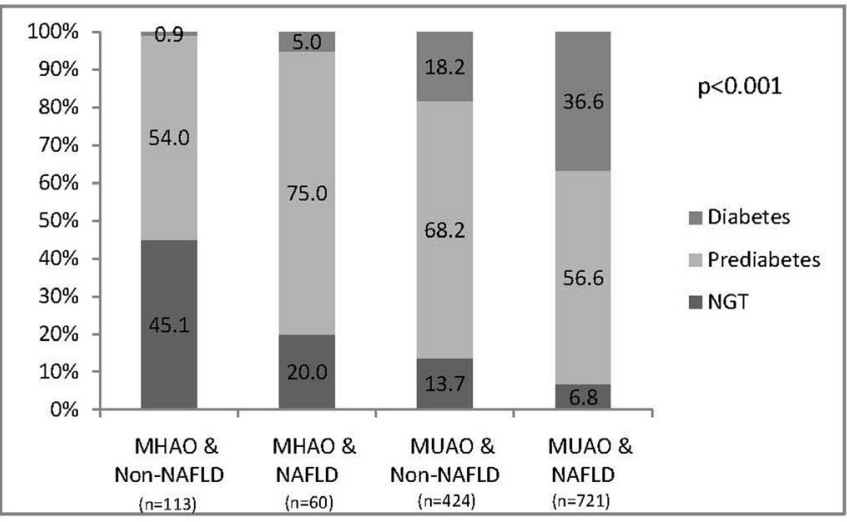

Figure 1 Prevalence rates (\%) of prediabetes and diabetes stratified by MHAO and NAFLD. MHAO, metabolically healthy abdominal obesity; MUAO, metabolically unhealthy abdominal obesity; NAFLD, non-alcoholic fatty liver disease; NGT, normal glucose test.

1 , and the adjusted ORs (95\% CIs) were 4.81 (3.28 to 7.07, $\mathrm{p}<0.001$ ) and 2.93 (2.06 to $4.16, \mathrm{p}<0.001$ ), respectively. Compared with those MHAO and non-NAFLD, those with MHAO and NAFLD, MUAO and non-NAFLD, MUAO and NAFLD all showed significantly increased risks of prediabetes plus diabetes, and the adjusted ORs ( $95 \%$ CIs) were 3.30 ( 1.55 to $7.02, \mathrm{p}=0.002$ ), 4.59 (2.85 to $7.40, \mathrm{p}<0.001$ ) and 10.14 (6.17 to $16.66, \mathrm{p}<0.001)$, respectively. And there was a significantly positive trend between increasing categories jointly by MHAO with NAFLD (from MHAO and non-NAFLD, MHAO and NAFLD, MUAO and non-NAFLD to MUAO and NAFLD) and risk of prediabetes plus diabetes (trend test: $\mathrm{p}<0.001$ ). Further adjustment for other potential confounding factors in model 2 and model 3 did not change the results much. For all subjects, both FLI and HIS were significantly associated with risk of prediabetes plus diabetes with adjusted ORs (95\% CIs) of 1.02 (1.01 to 1.03) and 1.10 (1.05 to 1.15 ), respectively (both $p$ values $<0.05$, model 3). Stratified analyses showed that higher FLI and HIS were significantly associated with increased risk of prediabetes plus diabetes for subjects with MUAO only, but not for those with MHAO (model 3).

To explore if there were sexual differences on the associaitions of NAFLD and MHAO with diabetes or prediabetes plus diabetes, interaction tests of sex with NAFLD or MHAO on diabetes and prediabetes plus diabetes were conducted further in model 3. But all the interaction tests were not statistically significant (data not shown). Similarly, all the interaction tests among age group (if older than 50 years) with NAFLD or MHAO on diabetes or prediabetes plus diabetes were not statistically significant (data not shown).

\section{DISCUSSION}

The present study found that around 13\% of the 1318 community-living Chinese adults with abdominal obesity were identified as MHAO, and around $35 \%$ of these
MHAO were further diagnosed with NAFLD. The prevalence rates of diabetes were $0.9 \%, 5.0 \%, 18.2 \%$ and $36.6 \%$ for those with MHAO and non-NAFLD, MHAO and NAFLD, MUAO and non-NAFLD and MUAO and NAFLD, respectively. And the corresponding numbers of prediabetes plus diabetes were $54.9 \%, 80.0 \%, 86.3 \%$ and $93.2 \%$ accordingly. Multivariable logistic regression analyses with adjustment for potential confounding factors showed that both MUAO and NAFLD were significantly associated with increased risks of diabetes as well as prediabetes plus diabetes. Furthermore, compared with MHAO and non-NAFLD, MHAO and NAFLD also showed significantly increased risk for prediabetes plus diabetes. Additionally, there were significantly positive trends between increasing categories jointly by MHAO and NAFLD (from MHAO and non-NAFLD, MHAO and NAFLD, MUAO and non-NAFLD to MUAO and NAFLD) with risks of diabetes and prediabetes plus diabetes.

Obesity has been becoming a public health problem affecting around $20 \%$ of the general populations worldwide and its consequence, such as the associated increasing incidence of type 2 diabetes mellitus (T2DM), cardiovascular disease, chronic kidney disease and some kinds of cancers, has been well documented. ${ }^{28}{ }^{29}$ In the past decade, studies have identified a subgroup of obese populations named 'MHO' who are devoid of multiple metabolic risk factors, such as impaired glucose regulation, dyslipidemia and hypertension. However, there are still quite a few concerns on MHO which should be clarified, such as its definition, diagnosis criteria as well as its long-term stability. ${ }^{30-32}$ Whether MHO represents a benign condition and its risk on diabetes remains poorly understood and available evidence are still controversial. ${ }^{33-35}$ Based on the Whitehall II cohort study with 7122 participants and a median follow-up period of 17.4 years, Hinnouho et al found that $279(3.9 \%)$ were identified as $\mathrm{MHO}$ and that MHO showed a significant increased risk of T2DM incidence ( $\mathrm{HR}=3.25,95 \%$ CI 2.32 to 4.54$)$ compared with metabolically healthy normal weight as well as a significant decreased risk compared with metabolically unhealthy obesity (MUO) (HR=1.98 (MUO vs $\mathrm{MHO}), 95 \%$ CI 1.39 to 2.83$).{ }^{33}$ A population-based prospective cohort study with 3038 subject (179 (5.7\%) $\mathrm{MHO}$ ) at baseline and about 11 years of follow-up found that subjects with MHO were more likely to develop T2DM than metabolically healthy non-obesity (MHNO) (relative risk $(\mathrm{RR})=3.44,95 \% \mathrm{CI} 1.84$ to 6.43$).{ }^{34}$ It should be mentioned that MHO were diagnosed if two or fewer of the four criteria of metabolism syndrome were met for those subjects with obesity in the studies above. In the present study, we defined those with none of the criteria of metabolism syndrome was met as being metabolically healthy and we could only dichotomized subjects as MHAO versus MUAO since all of the subjects were abdominal obese. Similar to results from the Whitehall II cohort study, we found that MUAO showed significantly increased risk of T2DM than MHAO with the much higher adjusted OR of 9.40 (95\% CI 3.38 to 
Table 2 Adjusted ORs with associated 95\% Cls of joint MHAO with NAFLD for diabetes and prediabetes plus diabetes

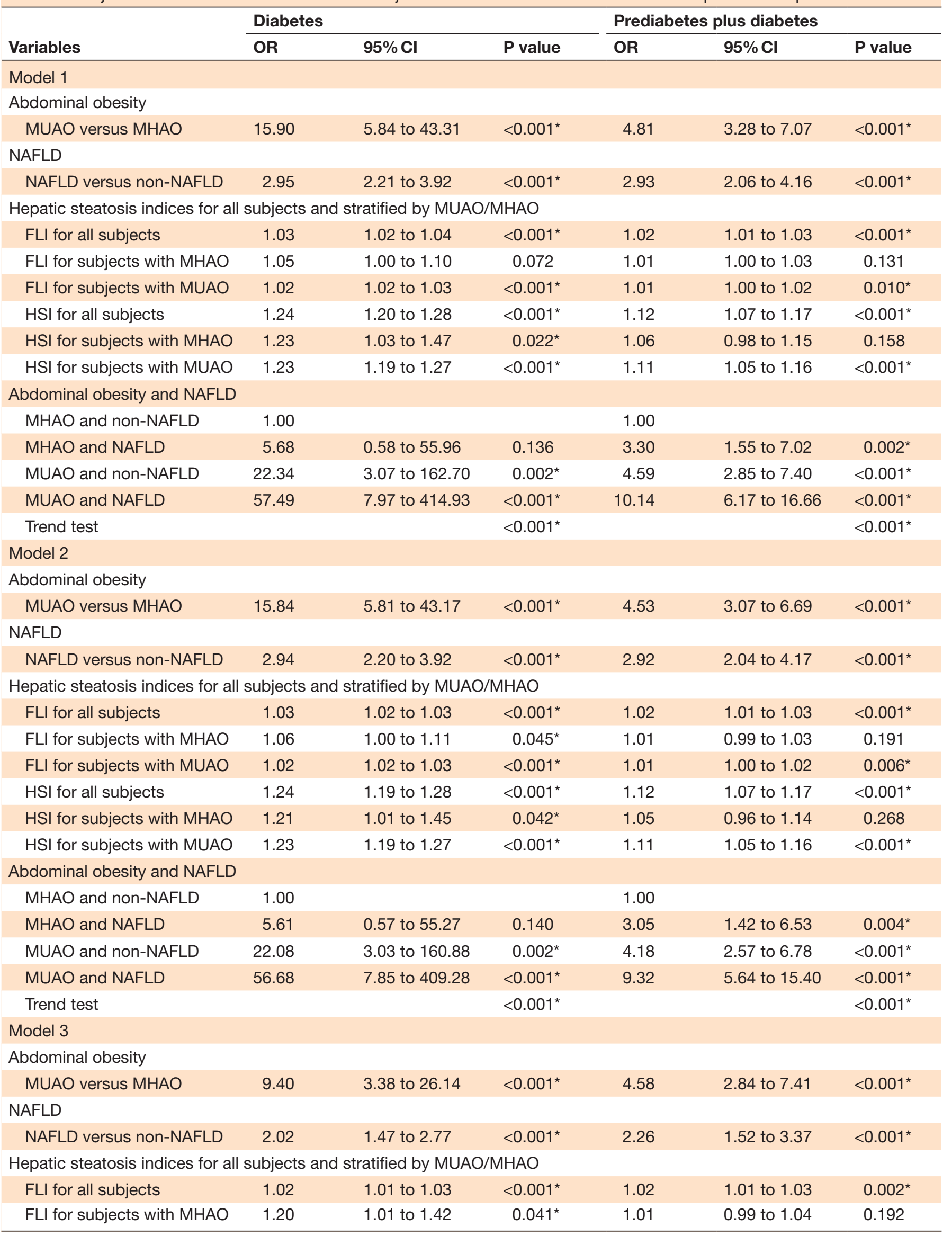


Table 2 Continued

\begin{tabular}{|c|c|c|c|c|c|c|}
\hline \multirow[b]{2}{*}{ Variables } & \multicolumn{3}{|c|}{ Diabetes } & \multicolumn{3}{|c|}{ Prediabetes plus diabetes } \\
\hline & OR & $95 \% \mathrm{Cl}$ & $P$ value & OR & $95 \% \mathrm{Cl}$ & $P$ value \\
\hline FLI for subjects with MUAO & 1.02 & 1.01 to 1.03 & $<0.001^{\star}$ & 1.01 & 1.00 to 1.03 & $0.021^{*}$ \\
\hline HSI for all subjects & 1.22 & 1.18 to 1.27 & $<0.001^{*}$ & 1.10 & 1.05 to 1.15 & $<0.001^{\star}$ \\
\hline HSI for subjects with MHAO & 1.31 & 1.01 to 1.70 & $0.039^{*}$ & 1.04 & 0.95 to 1.14 & 0.409 \\
\hline HSI for subjects with MUAO & 1.22 & 1.18 to 1.27 & $<0.001^{*}$ & 1.12 & 1.06 to 1.18 & $<0.001^{*}$ \\
\hline \multicolumn{7}{|l|}{ Abdominal obesity and NAFLD } \\
\hline MHAO and non-NAFLD & 1.00 & & & 1.00 & & \\
\hline MHAO and NAFLD & 5.10 & 0.52 to 50.30 & 0.163 & 2.87 & 1.32 to 6.27 & $0.008^{\star}$ \\
\hline MUAO and non-NAFLD & 17.02 & 2.32 to 124.98 & $0.005^{*}$ & 4.99 & 2.88 to 8.62 & $<0.001^{*}$ \\
\hline MUAO and NAFLD & 32.95 & 4.49 to 241.86 & $0.001^{*}$ & 10.17 & 5.41 to 19.11 & $<0.001^{\star}$ \\
\hline Trend test & & & $<0.001^{*}$ & & & $<0.001^{*}$ \\
\hline
\end{tabular}

Model 1 was adjusted for age and sex.

Model 2 was further adjusted for educational level, ever smoking, ever drinking and physical activity.

Model 3 was further adjusted for BMI, systolic and diastolic BP, triglyceride, total cholesterol, HDL-cholesterol and LDL-cholesterol and serum uric acid.

${ }^{*} \mathrm{P}<0.05$.

BMI, body mass index; BP, blood pressure; FLI, fatty liver index; HDL, high-density lipoprotein; HSI, hepatic steatosis index; LDL, low-density lipoprotein; MHAO, metabolically healthy abdominal obesity; MUAO, metabolically unhealthy abdominal obesity; NAFLD, non-alcoholic fatty liver disease.

26.14). We further found the excessive risks of MUAO, as compared with MHAO, on prediabetes plus diabetes $(\mathrm{RR}=4.58,95 \% \mathrm{CI} 2.84$ to 7.41$)$. Whether MHAO showed increased risk of T2DM compared with MHNO could not be assessed in the present study, since all of the subjects were abdominal obese, and we should conduct further studies to address this issue in future.

NAFLD has been consistently shown to be associated with metabolic/IR syndrome and thus has been proposed to predict T2DM. ${ }^{36}$ We previously found that

NAFLD was significantly associated with increased risk of T2DM prevalence. ${ }^{19}$ In the present study, we found that NAFLD was significantly associated with increased risk of T2DM which was consistent with others' studies ${ }^{36}$ and its significantly increased risk on prediabetes plus diabetes ( $R R=2.26$, 95\% CI 1.52 to 3.37). Furthermore, we found that higher hepatic steatosis indices, including FLI and HIS, were significantly associated with increased risks of diabetes and prediabetes plus diabetes. Our previous findings implied that NAFLD contributed to the pathogenesis of T2DM via multiple mechanisms besides its effect on metabolic/IR syndrome,$^{19}$ more underlying mechanisms linking NAFLD to T2DM incidence need further studies to clarify in future.

MHO has not been considered jointly with NAFLD to predicting the risk of diabetes. To the best of our knowledge, we are probably the first to explore the joint associations of MHAO with NAFLD on risks of diabetes and prediabetes. In the present study, we found that, compared with those MHAO and non-NAFLD, subjects with MUAO and non-NAFLD and MUAO and NAFLD showed significantly increased risks of diabetes as well as prediabetes plus diabetes, and those with MHAO and
NAFLD showed significantly excessive risk on prediabetes plus diabetes. Positive trends between increasing categories jointly by MHAO with NAFLD (from MHAO and non-NAFLD, MHAO and NAFLD, MUAO and nonNAFLD to MUAO and NAFLD) and risks of diabetes as well as prediabetes plus diabetes account for other novel findings in the present study. Moreover, stratified analyses in the present study showed that both increased FLI and HSI were significantly associated with elevated risk of diabetes even for those subjects with MHAO. The ideas, definitions and diagnosis criteria of $\mathrm{MHO}$ are still in debating. Fat accumulation in liver is usually not considered for all indices of general and abdominal obesity, and consequently NAFLD has not been included in the criteria of MHO. We found that about $35 \%$ of subjects with MHAO were further diagnosed with NAFLD, and subjects with MHAO and NAFLD showed significantly increased risk of prediabetes plus diabetes than those with MHAO and non-NAFLD. Our findings implied that MHAO based on the current diagnosis criteria was not really 'healthy', therefore NAFLD should be considered as one more potential criterion when defining MHO if more evidence could prove our findings in future, especially from the prospective cohort studies with larger sample sizes.

Literature has demonstrated that both NAFLD and diabetes have sexual differences. ${ }^{37}{ }^{38}$ We therefore explored if there were significant sex differences on the associations of NAFLD and MHAO with diabetes or prediabetes plus diabetes. Unfortunately, all the interactions between sex and NAFLD or MHAO were not statistically significant. Furthermore, to explore if age or menopausal status modified the associations of NAFLD and MHAO with diabetes, we treated 
age $\geq 50$ as a surrogate for menopause. Similarly, all the interaction terms between age group (if older than 50 years) and NAFLD or MHAO on the associations with diabetes or prediabetes plus diabetes were not statistically significant. We must acknowledge that the present sample size was relatively small and we may not have enough power to find the potential sex or age difference on these associations. Therefore, future studies with much larger sample size, especially in prospective cohort study design, are warranted to explore the potential sex or age difference on these associations.

We should be cautious when interpreting our results due to the following limitations of the present study. First, all subjects were abdominal obesity and were not randomly sampled from their living communities; therefore referring to the risk of diabetes and prediabetes, we could not assess the effect of MHAO as compared with MHNO and we might also underestimate the true joint associations of MHAO with NAFLD on diabetes. Second, NAFLD was determined by hepatic ultrasonography scanning in 2011, we had only data on description of hepatic steatosis diagnoses but did not have data on semiquantitative indices of liver ultrasonography as present. Therefore, future studies with more accurate and severities of NAFLD by hepatic ultrasonography scanning are needed. Third, as the present study was based on the baseline information of our ongoing cohort study, we cannot determine the temporal sequence among MHAO, NAFLD and T2DM. Future studies with longterm follow-up and information on diabetes incidence could be useful to address the issue.

\section{CONCLUSIONS}

NAFLD has not been considered in current definition and diagnosis criteria of $\mathrm{MHO}$, although liver is one of the main parts of fat accumulation when obesity occurs. The present study was probably the first to explore the joint associations of MHAO with NAFLD on risks of diabetes and prediabetes. We found that about $35 \%$ of subjects with MHAO accompanied by NAFLD showed significantly excessive risk of prediabetes plus diabetes compared with those MHAO and non-NAFLD. Furthermore, there were significantly positive trends between increasing categories jointly by MUAO with NAFLD and risks of diabetes as well as prediabetes plus diabetes. Stratified analyses showed that higher hepatic steatosis indices, including FLI and HIS, were significantly associated with increased risk of diabetes even for those subjects with MHAO. Therefore, our findings imply that NAFLD should be considered as one more criterion when defining and diagnosing MHO. Even for those seemly healthy obese, screening and intervention of NAFLD should be strengthened from the perspective of T2DM prevention.

\section{Author affiliations}

${ }^{1}$ Lianqian Community Health Service Center, The First Affiliated Hospital of Xiamen University, Xiamen, Fujian, China

${ }^{2}$ Department of Endocrinology, Zhongshan Hospital Fudan University Xiamen Branch, Xiamen, Fujian, China
${ }^{3}$ Department of Endocrinology and Diabetes, The First Affiliated Hospital of Xiamen University, Xiamen, Fujian, China

${ }^{4}$ Department of Nursing, The First Affiliated Hospital of Xiamen University, Xiamen, Fujian, China

${ }^{5}$ Xiamen Institute of Cardiovascular Diseases, The First Affiliated Hospital of Xiamen University, Xiamen, Fujian, China

${ }^{6}$ Key laboratory of Neonatal Diseases, Children's Hospital of Fudan University Xiamen Branch, Xiamen, Fujian, China

${ }^{7}$ Epidemiology Research Unit, Translational Medicine Research Center, The First Affiliated Hospital of Xiamen University, Xiamen, Fujian, China

Acknowledgements We are grateful to all the patients for their participation.

Contributors JZ, QX, FL and ZL performed the statistical analysis and wrote the manuscript; $\mathrm{ML}$ and YL participated in the data collection; SW, WZ and CL contributed to discussion; ZL, NC, SW and JZ participated in the design of the study and edited the manuscript. SW, NC and ZL are the guarantors of this work and, as such, had full access to all the data in the study and take responsibility for the integrity of the data and the accuracy of the data analysis.

Funding This study was supported by the grants from National Key R\&D Program of China (No. 2017YFC0907100) and Natural Science Foundation of China (No. 81870611).

Competing interests None declared.

Patient consent for publication Not required.

Ethics approval This study was approved by the Human Research Ethics Committee of the First Affiliated Hospital of Xiamen University (Xiamen, China) (number/ID of the obtained ethics approval: 2011YLS-013) and conducted according to the principles of the Declaration of Helsinki. Written informed consent was obtained from each participant.

Provenance and peer review Not commissioned; externally peer reviewed.

Data availability statement Data are available upon reasonable request. Data are available from the corresponding author upon reasonable request.

Open access This is an open access article distributed in accordance with the Creative Commons Attribution Non Commercial (CC BY-NC 4.0) license, which permits others to distribute, remix, adapt, build upon this work non-commercially, and license their derivative works on different terms, provided the original work is properly cited, appropriate credit is given, any changes made indicated, and the use is non-commercial. See: http://creativecommons.org/licenses/by-nc/4.0/.

\section{ORCID iDs}

Changqin Liu http://orcid.org/0000-0001-8063-7906

Zhibin Li http://orcid.org/0000-0001-6642-9780

\section{REFERENCES}

1 Zheng Y, Ley SH, Hu FB. Global aetiology and epidemiology of type 2 diabetes mellitus and its complications. Nat Rev Endocrinol 2018;14:88-98.

2 Saeedi P, Petersohn I, Salpea P, et al. Global and regional diabetes prevalence estimates for 2019 and projections for 2030 and 2045: Results from the International Diabetes Federation Diabetes Atlas, $9^{\text {th }}$ edition. Diabetes Res Clin Pract 2019;157:107843.

3 Li Y, Teng D, Shi X, et al. Prevalence of diabetes recorded in mainland China using 2018 diagnostic criteria from the American diabetes association: national cross sectional study. BMJ 2020;369:m997.

4 Tobias DK, Pan A, Jackson CL, et al. Body-Mass index and mortality among adults with incident type 2 diabetes. N Engl J Med 2014;370:233-44.

5 Berrington de Gonzalez A, Hartge P, Cerhan JR, et al. Body-Mass index and mortality among 1.46 million white adults. N Engl J Med 2010;363:2211-9.

6 Prospective Studies Collaboration, Whitlock G, Lewington S, et al. Body-Mass index and cause-specific mortality in 900000 adults: collaborative analyses of 57 prospective studies. Lancet 2009;373:1083-96

7 VAGUE J. The degree of masculine differentiation of obesities: a factor determining predisposition to diabetes, atherosclerosis, gout, and uric calculous disease. Am J Clin Nutr 1956;4:20-34.

8 Sims EA. Are there persons who are obese, but metabolically healthy? Metabolism 2001;50:1499-504. 
9 Stefan N, Kantartzis K, Machann J, et al. Identification and characterization of metabolically benign obesity in humans. Arch Intern Med 2008;168:1609-16.

10 Chatterjee S, Khunti K, Davies MJ. Type 2 diabetes. Lancet 2017;389:2239-51.

11 Blüher M. Metabolically healthy obesity. Endocr Rev 2020;41:405-20.

12 Bell JA, Kivimaki M, Hamer M. Metabolically healthy obesity and risk of incident type 2 diabetes: a meta-analysis of prospective cohort studies. Obes Rev 2014;15:504-15.

13 Angulo P. Nonalcoholic fatty liver disease. N Engl J Med 2002;346:1221-31.

14 Targher G, Byrne CD. Clinical review: nonalcoholic fatty liver disease: a novel cardiometabolic risk factor for type 2 diabetes and its complications. J Clin Endocrinol Metab 2013;98:483-95.

15 Yki-Järvinen $\mathrm{H}$. Non-Alcoholic fatty liver disease as a cause and a consequence of metabolic syndrome. Lancet Diabetes Endocrinol 2014;2:901-10.

16 Musso G, Gambino R, Bo S, et al. Should nonalcoholic fatty liver disease be included in the definition of metabolic syndrome? A cross-sectional comparison with adult treatment panel III criteria in nonobese nondiabetic subjects. Diabetes Care 2008;31:562-8.

17 Lonardo A, Mantovani A, Lugari S, et al. Epidemiology and pathophysiology of the association between NAFLD and metabolically healthy or metabolically unhealthy obesity. Ann Hepatol 2020;19:359-66.

18 Yan B, Shi X, Zhang H, et al. Association of serum irisin with metabolic syndrome in obese Chinese adults. PLoS One 2014:9:e94235.

19 Li Z, Lin M, Liu C, et al. Fetuin-B links nonalcoholic fatty liver disease to type 2 diabetes via inducing insulin resistance: association and path analyses. Cytokine 2018;108:145-50.

20 Ascaso JF, Pardo S, Real JT, et al. Diagnosing insulin resistance by simple quantitative methods in subjects with normal glucose metabolism. Diabetes Care 2003;26:3320-5.

21 Zeng MD, Fan JG, Lu LG, et al. Guidelines for the diagnosis and treatment of nonalcoholic fatty liver diseases. J Dig Dis 2008;9:108-12.

22 Bedogni G, Bellentani S, Miglioli L, et al. The fatty liver index: a simple and accurate predictor of hepatic steatosis in the general population. BMC Gastroenterol 2006;6:33

23 Lee J-H, Kim D, Kim HJ, et al. Hepatic steatosis index: a simple screening tool reflecting nonalcoholic fatty liver disease. Dig Liver Dis 2010;42:503-8.

24 Zhou B-F, Cooperative Meta-Analysis Group of the Working Group on Obesity in China. Predictive values of body mass index and waist circumference for risk factors of certain related diseases in Chinese adults--study on optimal cut-off points of body mass index and waist circumference in Chinese adults. Biomed Environ Sci 2002;15:83-96

25 Alberti KGMM, Zimmet P, Shaw J, et al. The metabolic syndrome--a new worldwide definition. Lancet 2005;366:1059-62.

26 Alberti KGMM, Eckel RH, Grundy SM, et al. Harmonizing the metabolic syndrome: a joint interim statement of the International diabetes Federation Task force on epidemiology and prevention; National heart, lung, and blood Institute; American heart association; world heart Federation; international atherosclerosis Society; and international association for the study of obesity.. Circulation 2009;120:1640-5.

27 American Diabetes Association. 2. Classification and Diagnosis of Diabetes: Standards of Medical Care in Diabetes-2020. Diabetes Care 2020;43:S14-31.

28 Malik VS, Willett WC, Hu FB. Global obesity: trends, risk factors and policy implications. Nat Rev Endocrinol 2013;9:13-27.

29 GBD 2015 Obesity Collaborators, Afshin A, Forouzanfar MH, et al. Health effects of overweight and obesity in 195 countries over 25 years. N Engl J Med 2017;377:13-27.

30 Stefan N, Häring H-U, Hu FB, et al. Metabolically healthy obesity: epidemiology, mechanisms, and clinical implications. Lancet Diabetes Endocrinol 2013;1:152-62.

31 Blüher M. Are metabolically healthy obese individuals really healthy? Eur J Endocrinol 2014;171:R209-19.

32 Stefan N, Häring H-U, Schulze MB. Metabolically healthy obesity: the low-hanging fruit in obesity treatment? Lancet Diabetes Endocrinol 2018;6:249-58.

33 Hinnouho G-M, Czernichow S, Dugravot A, et al. Metabolically healthy obesity and the risk of cardiovascular disease and type 2 diabetes: the Whitehall II cohort study. Eur Heart J 2015;36:551-9.

34 Fingeret M, Marques-Vidal P, Vollenweider P. Incidence of type 2 diabetes, hypertension, and dyslipidemia in metabolically healthy obese and non-obese. Nutr Metab Cardiovasc Dis 2018;28:1036-44.

35 Tajik S, Mirzababaei A, Ghaedi E, et al. Risk of type 2 diabetes in metabolically healthy people in different categories of body mass index: an updated network meta-analysis of prospective cohort studies. J Cardiovasc Thorac Res 2019;11:254-63.

36 Mantovani A, Petracca G, Beatrice G, et al. Non-Alcoholic fatty liver disease and risk of incident diabetes mellitus: an updated metaanalysis of 501022 adult individuals. Gut 2021;70:962-9.

37 Balakrishnan M, Patel P, Dunn-Valadez S, et al. Women have a lower risk of nonalcoholic fatty liver disease but a higher risk of progression vs men: a systematic review and meta-analysis. Clin Gastroenterol Hepatol 2021;19:61-71.

38 Lonardo A, Nascimbeni F, Ballestri S, et al. Sex differences in nonalcoholic fatty liver disease: state of the art and identification of research gaps. Hepatology 2019;70:1457-69. 